\title{
Effets des sels minéraux sur l'activité de la lipoprotéine lipase du lait de vache in vitro
}

\author{
Y Hannan, D Le Goff, L Friloux, A Nouvelot *
}

IRBA, URA 609 CNRS, laboratoire de biochimie, esplanade de la Paix, 14032 Caen Cedex, France

(Reçu le 10 juin 1988; accepté le 25 octobre 1989)

\begin{abstract}
Résumé - Les effets de 4 éléments minéraux présents dans le lait $\left(\mathrm{Na}^{+}, \mathrm{K}+, \mathrm{Ca}^{++}\right.$et $\left.\mathrm{Mg}++\right)$ ont été étudiés sur l'activité in vitro de la lipoprotéine lipase présente dans le lait écrémé (mLPL) et de la LPL purifiée du lait (LPL) en présence ou non d'activateur lipoprotéinique d'origine sérique (HDL), afin de mettre en évidence l'incidence de ces facteurs sur l'intensité de la lipolyse et la régulation de l'enzyme lipase. Les résultats obtenus montrent une très grande similitude de comportement de la lipoprotéine lipase du lait écrémé ou sous forme purifiée. La présence d'HDL dans le milieu d'incubation augmente de 7 fois l'activité de la mLPL et de 5 fois l'activité de la LPL purifiée. De tous les éléments minéraux étudiés, ce sont les ions divalents qui ont le plus fort pouvoir inhibiteur sur l'activité de la lipoprotéine lipase. Cet effet inhibiteur, modulé par la présence de lipoprotéines de haute densité (HDL), dépend essentiellement de la concentration et de la nature des ions ajoutés dans le milieu et non de la force ionique. A I = 0,24 l'inhibition de la LPL par $\mathrm{CaCl}_{2}$ est de $75 \%$ en présence d'HDL, et de $90 \%$ en son absence. En revanche, à la même force ionique et en présence de $\mathrm{NaCl}$, l'activité de la LPL n'est pas modifiée en présence ou en absence d'HDL dans le milieu d'incubation.
\end{abstract}

lipoprotéine lipase / lait de vache / lipoprotéine HDL / minéral

Summary - Effects of mineral salts on the activity of the bovine milk lipoprotein lipase in vitro. The effects of some milk constituents such as mineral elements $\left(\mathrm{Na}^{+}, \mathrm{K}^{+}, \mathrm{Ca}^{++}\right.$and $\left.\mathrm{Mg}^{++}\right)$have been studied in vitro on the $m L P L$ (skim milk) activity and that of the purified LPL, in presence and in absence of serum lipoprotein (HDL), with a view to highlighting the incidence of these factors on the lipolysis intensity and the regulation of the enzyme lipase through these factors. The results have shown a clear similarity of the LPL behaviour of skim milk or in a purified form. The presence of HDL in the incubation medium increases seven times the $m L P L$ activity and five times the purified $L P L$ activity. Amongst all mineral constituents involved in this study, divalent ions undoubtedly hold the highest inhibitory power of the lipoprotein lipase activity. This inhibitory effect, which has been modulated by the presence of HDL, depends mainly upon the concentration and the nature of ions added to the medium, but by no means upon the ionic strengh. With $I=0.24$, the inhibition of the LPL by $\mathrm{CaCl}_{2}$ is $75 \%$ in presence of $\mathrm{HDL}$ and $90 \%$ without $\mathrm{HDL}$ in the incubation medium. Contrastingly, with a similar ionic strengh and in presence of $\mathrm{NaCl}$, this activity is subject to any modification neither in presence nor in absence of HDL in the incubation medium.

lipoprotein lipase / bovine milk / lipoprotein HDL / mineral

\footnotetext{
* Correspondance et tirés à part
} 


\section{INTRODUCTION}

L'intensité de la lipolyse du lait en absence d'activation mécanique ou thermique par hydrolyse enzymatique de la matière grasse présente une très grande variabilité. De nombreux facteurs ont été impliqués dans la régulation de l'activité de la lipoprotéine lipase responsable de cette dégradation. Parmi les facteurs physiologiques les plus souvent évoqués, on peut citer le stade de lactation et de gestation, le niveau de production (Ortiz et al, 1970; Ahrné et Björck, 1985; Chazal et Chilliard, 1987a; Sundheim, 1988), la race dont l'incidence est beaucoup controversée (Chazal et Chilliard, 1987b). On peut noter également le rôle de la distribution de la $\mathrm{mLPL}$ dans les différentes fractions du lait (Wang et Randolph, 1978; Sundheim et Bengtsson-Olivecrona, 1985) ainsi que l'état de la membrane des globules gras qui bloque plus ou moins l'accès de l'enzyme vers son substrat (Sundheim et BengtssonOlivecrona, 1987b).

Parmi les facteurs physico-chimiques impliqués dans cette régulation, on peut citer le changement de température (Sundheim et Bengtsson-Olivecrona, 1987a), l'agitation mécanique (Needs et al, 1985), le taux de protéose peptone dans le lait (Anderson, 1981; Cartier et Chilliard, 1986), l'état d'association de l'enzyme avec la caséine ou de dissociation par l'héparine (Anderson, 1982), la quantité d'activateurs de la $L P L$ telles les lipoprotéines HDL (Anderson, 1979; Sundheim et al, 1983) et enfin la concentration en éléments minéraux (Shahani et Chandan, 1965). Ces derniers ont fait l'objet de nombreux travaux quant à leur incidence sur l'activité de la LPL purifiée, notamment en ce qui concerne le sodium (Egelrud et Olivecrona, 1973; Driessen et Stadhouders, 1974; Bengtsson et Olivecrona, 1982). En revanche, les études portant sur l'incidence d'autres éléments minéraux du lait sur l'activité de la LPL sont moins bien documentées.

L'objet de ce travail porte sur les effets spécifiques des 4 principaux éléments minéraux du lait, 2 monovalents $\left(\mathrm{Na}^{+}\right.$et $\mathrm{K}+$ ) et 2 divalents $\left(\mathrm{Mg}^{++}\right.$et $\left.\mathrm{Ca}^{++}\right)$, sur l'activité in vitro de la lipoprotéine lipase présente dans le lait écrémé (mLPL) et de la lipoprotéine lipase purifiée (LPL) en présence ou en absence d'HDL.

\section{MATÉRIEL ET MÉTHODES}

\section{Prélèvement de lait}

Le lait utilisé est constitué par un prélèvement de 2 I d'un mélange provenant de la traite du soir de 10 vaches de race normande. Aussitôt après la prise d'échantillon, le lait est centrifugé à $3000 \mathrm{~g}$ à $4{ }^{\circ} \mathrm{C}$ pendant $30 \mathrm{~min}$. Le lait écrémé contenant la lipoprotéine lipase ( $\mathrm{mLPL}$ ) est divisé en petites fractions de $2 \mathrm{ml}$ et congelé à $-20^{\circ} \mathrm{C}$.

\section{Préparation de la LPL purifiée}

La LPL est purifiée à partir du lait écrémé par préparation de poudre acétone-diéthyl éther après adsorption de l'enzyme sur les globules gras de I'Intralipid (Vitrum, Stockholm, Suède), selon la technique décrite par Posner et al (1983). Quinze mg de poudre sont dissous dans $4 \mathrm{ml}$ d'un tampon $50 \mathrm{mM} \mathrm{NH}{ }_{4} \mathrm{OH}-\mathrm{HCl}, \mathrm{pH} 8,5$ contenant $0,1 \%$ de Triton $\mathrm{X}-100$ (v/v) (Sigma). La solution est centrifugée pendant $4 \mathrm{~min}$ à faible vitesse (1 $500 \mathrm{~g})$. La LPL contenue dans le surnageant est purifiée par chromatographie d'affinité sur une colonne de sépharosehéparine $4 \mathrm{~B}$ préparée selon la technique décrite par Iverius (1971). Compte tenu de la très grande instabilité de l'enzyme purifiée, la préparation obtenue est immédiatement utilisée pour les différentes études cinétiques. 


\section{Conditions expérimentales des études enzymatiques}

Le substrat utilisé est constitué par $250 \mathrm{mg}$ de trioléoylglycérol purifié par CCM (Sigma) et de $10 \mu \mathrm{Ci}$ de Tri [1-14C] oléoylglycérol $(51 \mathrm{mCi} /$ mmol - Amersham) dans $20 \mathrm{ml}$ de tampon Tris$\mathrm{HCl} 0,2 \mathrm{M}$ à pH 8,5 contenant $6 \%$ de sérum albumine bovine (free fatty acid fraction, Sigma) et $0,1 \%(\mathrm{~V} / \mathrm{v})$ de Triton X-100 (Sigma). Le mélange est ensuite émulsionné par ultrasons pendant 10 min dans un bain de glace.

Le milieu d'incubation préparé selon le protocole décrit par Nilsson-Ehle et Schotz (1976) contient le substrat à la concentration de 5,6 $\mathrm{mM}$ et la source d'enzyme provenant soit de lait écrémé $(24 \mu \mathrm{l} / \mathrm{ml})$, soit de la LPL purifiée $(0,3$ $\mu \mathrm{g} / \mathrm{ml})$. A ce milieu standard sont ajoutés, selon les cinétiques étudiées, l'une ou l'autre des solutions salines $\left(\mathrm{KCl}, \mathrm{NaCl}, \mathrm{MgCl}_{2}\right.$ ou $\left.\mathrm{CaCl}_{2}\right)$ et l'activateur lipoprotéinique (HDL) à raison de $750 \mu \mathrm{g}$ de protéine/ml. Les concentrations en sels des différentes solutions ajoutées au milieu standard sont établies de façon à ce que l'augmentation de force ionique du milieu soit la même pour chaque élément minéral utilisé. Dans les études sur la LPL purifiée, seuls les sels de sodium et de calcium ont été testés.

Après incubation pendant $30 \mathrm{~min}$ à $37^{\circ} \mathrm{C}$ en présence d'HDL ou 120 min à $37^{\circ} \mathrm{C}$ en absence d'HDL, la réaction est stoppée par addition de $3,25 \mathrm{ml}$ d'un mélange $\mathrm{CHCl}_{3}, \mathrm{C}_{7} \mathrm{H}_{16}, \mathrm{CH}_{3} \mathrm{OH}$ $(5: 4: 5,6, v / v / v)$ (Belfrage et Vaughan, 1969) et 1 $\mathrm{ml}$ de $\mathrm{NaOH}$ 0,2 M (Wang et al, 1985). Le mélange est agité au vortex pendant $30 \mathrm{~s}$ puis centrifugé à $3000 \mathrm{~g}$ pendant $15 \mathrm{~min}$. La radioactivité est mesurée sur $1 \mathrm{ml}$ de la phase supérieure mélangée à $4 \mathrm{ml}$ de liquide de scintillation (Picofluor-Packard).

L'activité spécifique est exprimée en $\mu$ moles d'acide oléique libérées par min et par $\mathrm{mg}$ de protéine. La concentration protéique est déterminée selon la méthode de Lowry et al (1951).

La force ionique est calculée par l'équation :

$$
I=1 / 2 \Sigma M i Z i 2
$$

Mi étant la molarité des ions et Zi la charge des ions (Segel, 1976).

\section{Préparation des HDL}

Les HDL sont préparées à partir du sérum de vache par ultracentrifugation séquentielle selon la méthode décrite par Socorro et Camejo (1979). Les limites de densité retenues pour isoler les HDL bovines sont comprises entre 1,05 et $1,1 \mathrm{~g} / \mathrm{ml}$ (Quincey et al, 1987). La fraction HDL est ensuite dialysée pendant $72 \mathrm{~h}$ contre une solution à $0,01 \%$ de $\mathrm{NaN}_{3}$ (Sigma) et $0,04 \%$ de $\mathrm{Na}_{2}$ EDTA (Merck) à $4^{\circ} \mathrm{C}$, puis stockée à $-30^{\circ} \mathrm{C}$.

\section{RÉSULTATS}

\section{Action des lipoprotéines de haute den- sité (HDL) sur la lipoprotéine lipase}

La relation entre le temps d'incubation et l'activité de l'enzyme lipase a été étudiée pendant $30 \mathrm{~min}$ en présence d'activateur HDL et 120 min en son absence. Dans nos conditions expérimentales, quelle que soit la source de lipoprotéine lipase (mLPL ou LPL purifiée), la relation est linéaire jusqu'à $35 \mathrm{~min}$ en présence d'HDL et $140 \mathrm{~min}$ en absence d'HDL. La présence d'HDL dans le milieu d'incubation augmente l'activité spécifique de la $\mathrm{mLPL}: 9 \mathrm{nmol} / \mathrm{min} / \mathrm{mg}$ de protéines en absence d'HDL contre 62 $\mathrm{nmol} / \mathrm{min} / \mathrm{mg}$ de protéines en présence d'HDL. Le même phénomène a été observé avec la LPL purifiée, l'activité spécifique passant de $39,5 \mu \mathrm{mol} / \mathrm{min} / \mathrm{mg}$ de protéines en absence d'HDL à $200 \mu \mathrm{mol} / \mathrm{min} / \mathrm{mg}$ de protéines en présence d'HDL.

\section{Effet du sodium et du potassium sur l'activité de la mLPL}

Les variations de l'activité de la $\mathrm{mLPL}$ en fonction des concentrations en $\mathrm{NaCl}$ et $\mathrm{KCl}$ 
sont reportées sur la figure 1. En présence d'HDL, on observe une inhibition de l'activité de la mLPL similaire pour les ions sodium et potassium. Cette inhibition atteint $38 \%$ lorsque ces 2 sels sont ajoutés à une concentration de $600 \mathrm{mM}$ dans le milieu. En absence d'HDL, cette inhibition n'est constatée qu'à partir d'une concentration en sel de sodium de $240 \mathrm{mM}$ et atteint $32 \%$ à $600 \mathrm{mM}$. L'absence de l'activateur ne modifie pas l'allure de la courbe d'inhibition mais diminue la sensibilité de l'enzyme. Pour les 2 sels étudiés, on obtient une courbe d'inhibition biphasique avec un point d'inflexion correspondant à des concentrations en sels comprises entre 240 et $360 \mathrm{mM}$.

\section{Effet du calcium et du magnésium sur l'activité de la mLPL}

L'addition de concentrations croissantes en calcium et magnésium induit une très nette inhibition de l'activité de la mLPL (fig 2). On peut constater d'une part que l'inhibition est plus marquée avec le calcium et d'autre part que lorsque les deux sels sont ajoutés à une concentration supérieure à $120 \mathrm{mM}$, l'activité résiduelle de la $\mathrm{mLPL}$ est nulle. Contrairement à ce que nous avons observé avec le sodium, l'absence d'HDL ne fait qu'accentuer l'intensité de l'inhibition. Ainsi, l'addition de calcium à 80 $\mathrm{mM}$ inhibe à $95 \%$ l'activité de la $\mathrm{mLPL}$ alors que l'addition de la même concentration d'ions $\mathrm{Na}+$ provoque une légère stimulation de la mLPL.

\section{Effet du calcium et du sodium sur la LPL purifiée}

L'activité résiduelle de l'enzyme lipase en fonction de la concentration de $\mathrm{CaCl}_{2}$ est présentée fig 3 en présence d'HDL et fig 4

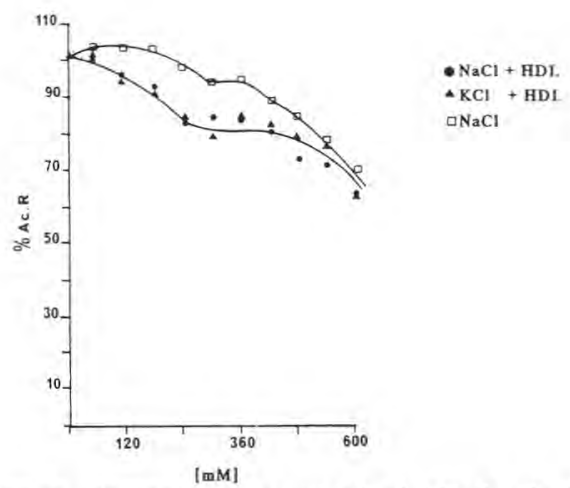

Fig 1. Pourcentage d'activité résiduelle de la $\mathrm{mLPL}$ en fonction de différentes concentrations de sels monovalents. $\mathrm{NaCl}+750 \mu \mathrm{g} / \mathrm{ml} \mathrm{HDL}$ (•); $\mathrm{KCl}+750 \mu \mathrm{g} / \mathrm{ml} \mathrm{HDL} \mathrm{( \Delta );} \mathrm{NaCl}$ sans $\mathrm{HDL}$ (घ).

Percentage of residual activity of $m L P L$ at different monovalent salt concentrations. $\mathrm{NaCl}+750$ $\mu \mathrm{g} / \mathrm{ml} \mathrm{HDL}(\bullet) ; \mathrm{KCl}+750 \mu \mathrm{g} / \mathrm{ml}(\mathbf{\Delta}) ; \mathrm{NaCl}$ without $H D L(\square)$.

en absence d'HDL. L'addition de quantités croissantes de sels de calcium induit une très nette inhibition de l'activité de la LPL purifiée. En présence d'HDL, une inhibition

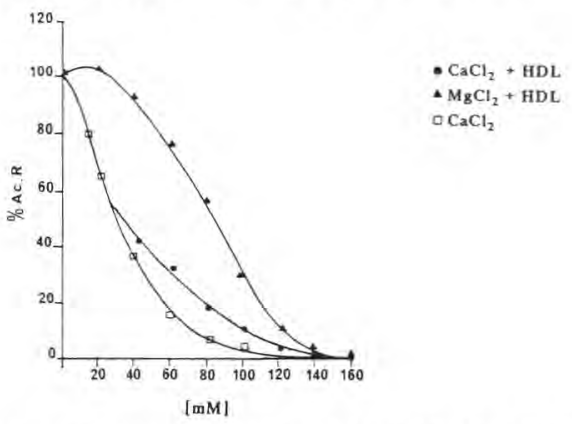

Fig 2. Pourcentage d'activité résiduelle de la $\mathrm{mLPL}$ en fonction de différentes concentrations de sels divalents. $\mathrm{CaCl}_{2}+750 \mu \mathrm{g} / \mathrm{ml} \mathrm{HDL}(\bullet)$; $\mathrm{MgCl}_{2}+750 \mu \mathrm{g} / \mathrm{ml} \mathrm{HDL}(\mathbf{\Delta}) ; \mathrm{CaCl}_{2}$ sans $\mathrm{HDL}$ (অ).

Percentage of residual activity of $M L P L$ at different divalent salt concentrations. $\mathrm{CaCl}_{2}+750 \mu \mathrm{g}$ $\mathrm{ml}(\Theta) ; \mathrm{MgCl}_{2}+750 \mu \mathrm{g} / \mathrm{ml}(\mathbf{\Delta}) ; \mathrm{CaCl}_{2}$ without $H D L(\square)$. 
complète est obtenue pour des concentrations en $\mathrm{CaCl}_{2}$ supérieures à $160 \mathrm{mM}$ (fig 3). En absence d'HDL, l'inhibition de la LPL par les ions calcium est accentuée. En effet, l'addition de $\mathrm{CaCl}_{2}$ à $80 \mathrm{mM}$ inhibe à 95\% l'activité de la LPL (fig 4).

L'activité résiduelle de la LPL purifiée en fonction du taux de sodium ajouté au milieu d'incubation est présentée dans les figures 3 et 4 . On constate un comportement très spécifique de la LPL vis-à-vis du chlorure de sodium. En effet, à faible concentration, on note une stimulation de l'activité en présence ou non d'HDL, alors qu'à forte concentration ( $\mathrm{NaCl} 360 \mathrm{mM}$ ), on observe une diminution de l'activité. L'inhibition par le $\mathrm{NaCl}$ reste toutefois partielle, puisqu'elle n'atteint que $40 \%$ en présence d'HDL et $50 \%$ en leur absence.

Le degré d'inhibition est indépendant de l'augmentation de force ionique du milieu.

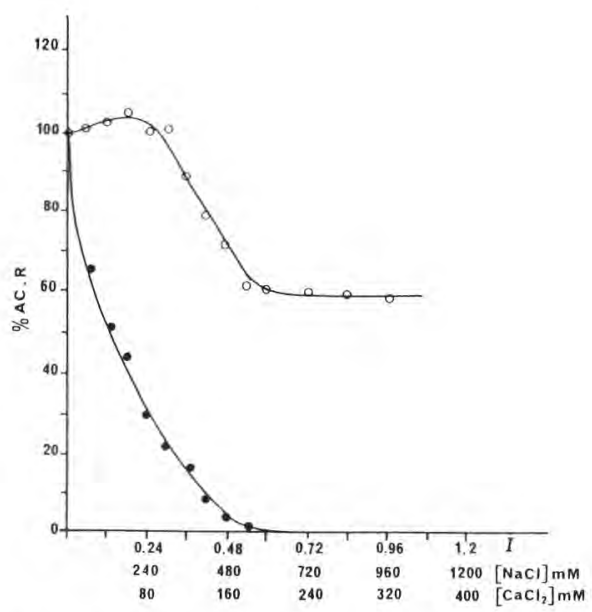

Fig 3. Pourcentage d'activité résiduelle de la LPL purifiée en fonction de la force ionique et des différentes concentrations en $\mathrm{CaCl}_{2}(\bullet)$ ou $\mathrm{NaCl}(O)$ en présence de $750 \mu \mathrm{g} / \mathrm{ml}$ de HDL.

Percentage of residual activity of purified LPL in function of ionic strength and different concentrations of $\mathrm{CaCl}_{2}(0)$ or $\mathrm{NaCl}(\mathrm{O})$ in presence of HDL $750 \mu \mathrm{g} / \mathrm{ml}$.
En effet pour une même élévation de force ionique $(I=0,24)$, on note une perte d'activité de $90 \%$ avec $\mathrm{CaCl}_{2}$ alors que l'activité n'est pas modifiée avec $\mathrm{NaCl}$.

\section{DISCUSSION}

L'augmentation du taux d'acides gras libres dans le lait par addition d'HDL est un phénomène bien connu (Clegg, 1980; Sundheim et al, 1983). Le taux d'HDL mais également leur répartition dans les différentes fractions du lait présenterait une très grande variabilité individuelle (Anderson, 1979). Selon cet auteur, la concentration en activateur d'un lait représente un facteur important de l'intensité de la lipolyse in vivo. Dans nos conditions in vitro, l'addition d'un taux saturant d'HDL augmente d'environ 7 fois le pouvoir catalyti-

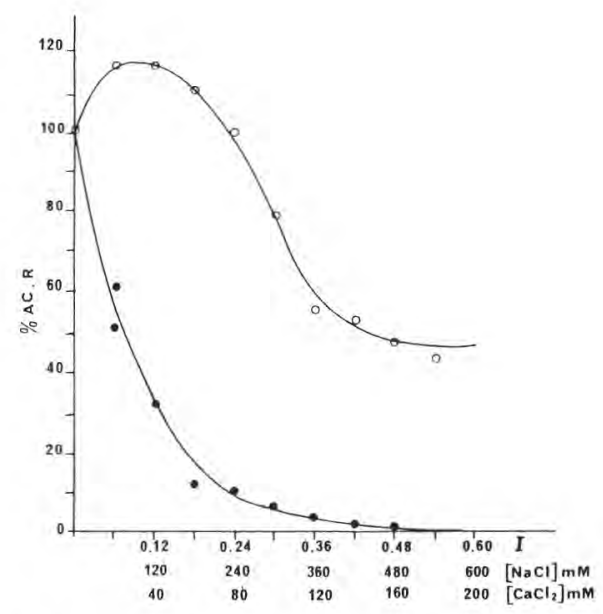

Fig. 4. Pourcentage d'activité résiduelle de la LPL purifiée en fonction de la force ionique et des différentes concentrations en $\mathrm{CaCl}_{2}(\bullet)$ ou $\mathrm{NaCl}(\mathrm{O})$ en absence de HDL.

Percentage of residual activity of purified LPL according to ionic strength and different concentrations of $\mathrm{CaCl}_{2}(\bullet)$ or $\mathrm{NaCl}(\mathrm{O})$ in absence of $H D L$. 
que de l'enzyme mLPL et d'environ 5 fois celui de l'enzyme LPL purifiée. Ainsi, nous avons pu confirmer une grande similitude de comportement de la mLPL et LPL purifiée. Toutefois, on peut noter que la stimulation de l'activité de la LPL par l'apoC-II vis-à-vis d'un substrat triglycéride est très variable suivant les auteurs et varie d'un facteur 2 à 30 (Posner, 1982).

Les ions monovalents ( $\mathrm{Na}^{+}, \mathrm{K}+$ ) induisent une perte d'activité de la mLPL tout à fait identique en présence d'HDL pour toutes les concentrations testées. L'activité de la mLPL ou sous forme purifiée en fonction de la concentration en sels monovalents évolue suivant une courbe biphasique. Aux concentrations inférieures à $0,25 \mathrm{M}$, on constate une légère stimulation de l'activité de la mLPL en absence d'HDL et en revanche, une perte de $15 \%$ d'activité de la mLPL en présence d'HDL. Aux concentrations supérieures à $0,25 \mathrm{M}$, on constate une forte inhibition de l'activité $\mathrm{mLPL}$ en présence ou en absence d'activateurs HDL. Fielding et Fielding (1976) ont montré que les sels de $\mathrm{Na} \mathrm{1,0} \mathrm{M} \mathrm{en-}$ traînent une inhibition de $70 \%$ de la LPL purifiée en présence de substrat trioléine et d'activateur VLDL. Dans nos conditions expérimentales (même substrat trioléine mais activateur HDL), I'inhibition de la LPL purifiée atteint $40 \%$ pour une concentration de $\mathrm{NaCl} 1 \mathrm{M}$. Cette différence peut être expliquée, en partie, par la nature différente de l'agent stabilisant (Riley et Robinson, 1974). L'effet $\mathrm{NaCl}$ sur l'activité de la LPL dépendrait non seulement de la concentration en sels mais également de la nature physique du substrat triacylglycérol. Ainsi, Rapp et Olivecrona (1978) décrivent une stimulation de la LPL purifiée en présence de substrat tributyrine et de sels de $\mathrm{Na} \mathrm{0,5} \mathrm{M}$. Plusieurs hypothèses ont été formulées quant au mode d'action du sodium sur la LPL purifiée. Les ions $\mathrm{Na}+$ pourraient intervenir au niveau du com- plexe enzyme-activateur (Fielding et Fielding, 1976), mais également au niveau du complexe enzyme-substrat (Posner et Morrisson, 1979). Nos résultats nous permettent de rejoindre cette dernière hypothèse selon laquelle le $\mathrm{NaCl}$, à faible concentration, augmente la liaison de l'enzyme avec le substrat et, à forte concentration, diminue l'interaction enzymesubstrat sans toutefois bloquer totalement son activité.

Pour toutes les concentrations testées, les ions divalents $\left(\mathrm{Ca}^{++}, \mathrm{Mg}^{++}\right)$exercent un effet inhibiteur sur la lipoprotéine lipase. Aux faibles concentrations, l'effet inhibiteur du calcium est beaucoup plus important que celui du magnésium. Cet effet inhibiteur, induit par les ions $\mathrm{Ca}^{++}$se manifeste dès les plus faibles concentrations en $\mathrm{CaCl}_{2}$ dans le milieu d'incubation mettant en évidence une grande sensibilité de la lipoprotéine lipase aux ions calcium. Cette très grande sensibilité est aussi observée sur la LPL purifiée. Les résultats obtenus par différents auteurs sur l'influence du calcium sur la LPL montrent une grande hétérogénéité. Korn et Quingley (1955) ont constaté qu'une forte concentration de $\mathrm{Ca}^{++}$inhibe l'activité de la LPL tissulaire mais qu'une faible concentration (10 à 20 $\mathrm{mM}$ ) augmente son activité, l'optimum de l'activité étant obtenu pour une concentration de $\mathrm{Ca}^{++} 10 \mathrm{mM}$. D'autres auteurs ont montré que le $\mathrm{Ca}++50 \mathrm{mM}$ stimule l'activité de la LPL posthéparine (Doizaki et Zieve, 1966; Posner et Morales, 1972). La sensibilité de l'enzyme dépendrait également de la nature des sels de calcium utilisés; ainsi, Shahani et Chandan (1965) ont montré que le pourcentage d'inhibition de la $\mathrm{LPL}$ purifiée était de $16 \%$ avec $\mathrm{CaCl}_{2}$ et de $45 \%$ avec $\mathrm{Ca}(\mathrm{OH})_{2}$ et ce pour des concentrations équivalentes en sels (5 $\mathrm{mM}$ ). Dans nos conditions, $50 \mathrm{mM}$ de $\mathrm{Ca}++$ produit une forte inhibition de la $\mathrm{mLPL}$ ou de la LPL purifiée et peut atteindre respec- 
tivement $62 \%$ et $75 \%$ en présence ou en absence d'HDL. Thomas et al (1971) considèrent que le $\mathrm{Ca}++$ agirait comme cofacteur de la LPL avec un optimum d'activité réalisé à la concentration physiologique de 1,25 à $2,5 \mathrm{mM}$. Au-delà de cette concentration, le $\mathrm{Ca}++$ inhiberait l'activité de la LPL tissulaire. L'hétérogénéité de ces résultats pourrait s'expliquer par les variations du taux de citrate dans la sérum albumine bovine présente dans le milieu d'incubation (Thomas et al, 1971) ou du type de détergent utilisé comme agent émulsifiant (Posner et Morales, 1972). Selon Thomas et al (1971), le Mg++ jusqu'à $12 \mathrm{mM}$ agit peu sur l'activité de la LPL tissulaire. Nos résultats, obtenus sur la $\mathrm{mLPL}$, sont en accord avec ceux de ces derniers auteurs.

Dans nos conditions expérimentales, l'intensité de l'inhibition observée in vitro sur la lipoprotéine lipase présente dans le lait écrémé ou sous forme purifiée dépend essentiellement de la nature des sels incorporés dans le milieu et non de la force ionique. On peut noter, cependant, que Fielding (1968) a rapporté que le taux d'inactivation de la LPL tissulaire était dépendant de la force ionique et non de la concentration des ions présents dans le milieu d'incubation.

Les résultats obtenus in vitro montrent une parfaite similitude de l'interdépendance de la lipoprotéine lipase du lait écrémé ou sous forme purifiée vis-à-vis des sels minéraux et d'HDL. Sans être directement extrapolables au comportement de la lipoprotéine lipase dans son milieu naturel, les résultats obtenus nous permettent de penser que la variabilité des concentrations en calcium et en lipoprotéines (HDL) dans le lait pourrait jouer un rôle dans les variations individuelles observées au niveau de la lipolyse du lait de vache.

\section{REMERCIEMENTS}

Nous remercions MC Dubourg pour la dactylographie du manuscrit. Cette étude a été réalisée grâce au concours financier du Conseil régional de Basse-Normandie.

\section{RÉFÉRENCES}

Ahrné L, Björck L (1985) Lipolysis and distribution of lipase activity in bovine milk in relation to stage of lactation and time of milking. $J$ Dairy Res 52, 55-64

Anderson M (1979) Enzyme immunoassay for measuring lipoprotein lipase activator in milk. J Dairy Sci 62, 1380-1383

Anderson M (1981) Inhibition of lipolysis in bovine milk by proteose peptone. J Dairy Res 48, 247-252

Anderson M (1982) Factors affecting the distribution of lipoprotein lipase activity between serum and casein micelles in bovine milk. $J$ Dairy Res 49, 51-59

Belfrage P, Vaughan M (1969) Simple liquidliquid partition system for isolation of labeled oleic acid from mixtures with glycerides. $J$ Lipid Res 10, 341-344

Bengtsson G, Olivecrona T (1982) The effects of $\mathrm{pH}$ and salt on the lipid binding and enzyme activity of lipoprotein lipase. Biochim Biophys Acta 751, 254-259

Cartier P, Chilliard $Y$ (1986) Effects of different skim milk fractions on activity of cow milk purified lipoprotein lipase. J Dairy Sci 69, 951955

Chazal MP, Chilliard Y (1987a) Les variations individuelles de la lipolyse spontanée du lait de vache : effet du numéro de lactation et répétabilité au cours de deux lactations successives. Lait 67, 437-450

Chazal MP, Chilliard Y (1987b) Effect of breed of cow (Friesian and Montbéliarde) on spontaneous and induced lipolysis in milk. J Dairy Res 54, 7-11

Clegg RA (1980) Activation of milk lipase by serum proteins: possible role in the occur- 
rence of lipolysis in the raw bovine milk. $J$ Dairy Res 47, 61-70

Doizaki WM, Zieve L (1966) An improved substrate preparation for postheparin plasma lipase. Proc Soc Exp Biol Med 122, 606-609

Driessen FM, Stadhouders J (1974) A study of spontaneous rancidity. Neth Milk Dairy $J 28$, 130-145

Egelrud T, Olivecrona T (1973) Purified bovine milk lipoprotein lipase: activity against lipid substrates in the absence of exogenous serum factors. Biochim Biophys Acta 306, 115-127

Fielding CJ (1968) Inactivation of lipoprotein lipase in buffered saline solution. Biochim Biophys Acta 159, 94-102

Fielding JC, Fielding EP (1976) Mechanism of salt-mediated inhibition of lipoprotein lipase. $J$ Lipid Res 17, 248-256

Iverius HP (1971) Coupling of glycosaminoglycans to agarose beads (sepharose 4B). Biochem J 124, 677-683

Korn ED, Quigley TW Jr (1955) Studies on lipoprotein lipase of rat heart and adipose tissue. Biochim Biophys Acta 18, 143-145

Lowry OH, Rosebrough NJ, Farr LA, Randall JR (1951) Protein measurement with the folin phenol reagent. J Biol Chem 193, 263-275

Needs CE, Anderson M, Payne JS, Ridout AE (1985) Influence of processing temperature and seasonal change in diet on lipase activity and lipolysis during the mechanical separation of bovine milk. $J$ Dairy Res 52,255 266

Nilsson-Ehle P, Schotz CM (1976) A stable radioactive substrate emulsion for assay of lipoprotein lipase. J Lipid Res 17, 536-541

Ortiz MJ, Keslere M, Watrous GH Jr, Cloninger WH (1970) Effect of the cow's body condition and stage of lactation on development of milk rancidity. J Milk Food Technol 33, 339342

Posner I, Morales A (1972) Mechanisms of enzyme and substrate activation by lipoprotein lipase cofactors. J Biol Chem 247, 22552265
Posner I, Morrisson DA (1979) The cofactor effects of serum lipoproteins on lipoproteins lipase activity and its inhibition by sodium chloride. Acta Cient Venez 30, 143-151

Posner I (1982) Mechanisms of enzyme activation in the lipoprotein lipase system. Atheroscler Rev 9, 123-156

Posner I, Wang CS, McConathy JW (1983) The comparative kinetics of soluble and immobilized bovine lipoprotein lipase. Arch Biochem Biophys 226, 306-318

Quincey D, Le Goff D, Fresnel J, Nouvelot A (1987) Quantitative alteration of bovine serum lipoproteins with ageing. Comp Biochem Physiol 88B, 929-937

Rapp D, Olivecrona T (1978) Kinetic of milk lipoprotein lipase. Studies with tributyrin. Eur $J$ Biochem 91, 379-385

Riley ES, Robinson SD (1974) Studies on the assay of clearing factor lipase (lipoprotein lipase). Biochim Biophys Acta 369, 371-386

Segel HI (1976) Biochemical Calculation. 2nd edition. Wiley J \& Sons, New York, 5

Shahani MK, Chandan CR (1965) Activity of purified milk lipase in the presence of milk constituents. Arch Biochem Biophys 111, 257-263

Socorro L, Camejo G (1979) Preparation and properties of soluble immuno-reactive Apo LDL. J Lipid Res 20, 631-638

Sundheim G, Zimmer LT, Astrup NH (1983) Induction of milk lipolysis by lipoprotein components of bovine blood serum. J Dairy Sci 66, 400-406

Sundheim G, Bengtsson-Olivecrona G (1985) Lipolysis in milk induced by cooling or by heparin: comparisons of amount of lipoprotein lipase in the cream fraction and degree of lipolysis. J Dairy Sci 68, 589-593

Sundheim G, Bengtsson-Olivecrona G (1987a) Isolated milk fat globules as substrate for lipoprotein lipase: study of factors relevant to spontaneous lipolysis in milk. J Dairy Sci 70, 499-505

Sundheim G, Bengtsson-Olivecrona G (1987b) Hydrolysis of bovine milk fat globules by lipoprotein lipase: inhibition by protein extracted 
from milk fat globule membrane. $J$ Dairy $S c i$ $70,1815-1821$

Sundheim G (1988) Spontaneous lipolysis in bovine milk: combined effects of cream, skim milk, and lipoprotein lipase activity. $J$ Dairy Sci 71, 620-626

Thomas F, Whayne Jr, Felts MJ (1971) Activation of lipoprotein lipase. Evaluation of cal- cium, magnesium, and ammonium as cofactors. Circ. Res. 28, 649-654

Wang CS, McConathy JW, Kloer UH, Alaupovic P (1985) Modulation of lipoprotein lipase activity by apolipoproteins. J Clin Invest 75, 384390

Wang L, Randolph EH (1978) Activation of lipolysis. I. Distribution of lipase activity in temperature activated milk. J Dairy Sci 61, 874-880 